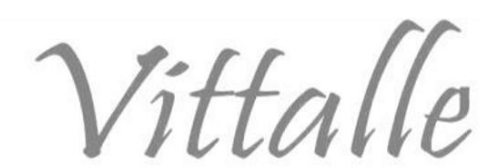

\title{
Terapias adicionais a plasmaférese como tratamento da púrpura trombocitopênica trombótica
}

\author{
Brunna Rafaela do Amaral Azevedo, Isabella de Souza Mota, \\ Milton Rego de Paula Junior ${ }^{*}$ \\ Centro Universitário de Brasília (UniCEUB), Brasília, DF, Brasil
}

\begin{abstract}
RESUMO
A púrpura trombocitopênica trombótica (PTT) é uma doença rara que possui caráter adquirido ou congênito, tendo como características intrínsecas a ambas a oclusão difusa da microcirculação por trombos, distúrbios neurológicos, trombocitopenia, disfunção renal e anemia hemolítica microangiopática. A fisiopatologia da PTT é ocasionada pela presença de multímeros de Fator de Von Willebrand (FvW) de alto peso molecular e deficiência da metaloprotease ADAMTS13. As terapias para o PTT variam entre a infusão de plasma fresco congelado (PFC) ou plasma de 24 horas para corrigir a deficiência da ADAMTS13, plasmaférese para retirada de dos multímeros e autoanticorpos anti-ADAMTS13, pulsoterapia com corticoides, utilização de anticorpos monoclonais quiméricos visando a diminuição da produção de autoanticorpos, e anticorpos específicos para FvW.
\end{abstract}

Additional therapies to plasmapheresis as treatment of thrombotic thrombocytopenic purpura

\section{ABSTRACT}

Thrombotic thrombocytopenic purpura (TTP) is a rare disease that has an acquired or congenital character, with intrinsic characteristics of both diffuse microcirculation occlusion by thrombus, neurological disorders, thrombocytopenia, renal dysfunction and microangiopathic hemolytic anemia. The pathophysiology of TPP is caused by the presence of high molecular weight Von Willebrand Factor (vWf) multimers and ADAMTS13 metalloprotease deficiency. TPP therapies range from infusion of fresh frozen plasma (FFP) or 24-hour plasma to correct ADAMTS13 deficiency, plasmapheresis to remove the multimers and anti-ADAMTS13 autoantibodies, pulse therapy with corticosteroids, use of chimeric monoclonal antibodies to decrease the production of autoantibodies, and specific antibodies to vWf.

\section{Introdução}

A púrpura trombocitopênica trombótica (PTT) é uma síndrome rara (5-10 casos/ 1.000.000/ ano) com maior incidência de casos em mulheres do que em homens (3:2), sendo predominante em adolescentes e jovens adultos (15-39 anos) $(1,2)$.

Descoberta em 1924 pelo médico Eli Moschcowitz, a PTT, também denominada como síndrome de Moschcowitz, possui caráter adquirido ou congênito, tendo como características intrínsecas em comum a oclusão difusa da microcirculação por trombos, distúrbios neurológicos, trombocitopenia, disfunção renal e anemia hemolítica microangiopática (3).

A fisiopatologia da PTT fora descrita por Joel Moake e colaboradores (4), onde foi

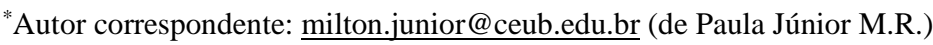


possível relacionar que em pacientes com episódios recorrentes, havia a presença de multímeros de Fator de Von Willebrand (FvW) de alto peso molecular, compreendendo que esses pacientes possuíam deficiência na clivagem desse polímero glicoproteico. $\mathrm{O}$ acúmulo de grandes multímeros de $\mathrm{FvW}(\mathrm{gmFvW})$ acarreta na agregação plaquetária, sendo a base dos sintomas envolvidos.

O fator de Von Willebrand (FvW) é uma glicoproteína cuja síntese ocorre através dos megacariócitos e das células endoteliais, sendo armazenado nos corpos de Weibel Palade e nos grânulos alfa das plaquetas. possuindo função de adesão plaquetária, também atuando impedindo a degradação proteolítica do FVIII. O gene que codifica a tradução da glicoproteína FvW está localizado no braço curto do cromossomo 12. Mutações nesse ponto ocasionam coagulopatia hereditária com disfunção plaquetária, tendo 30 a 100 casos/1.000.000 no mundo.

Diferente da maior parte dos fatores de coagulação, este não é produzido pelo fígado. Tem sua função ativada quando há presença de lesão endotelial, sendo necessária a modificação da sua estrutura, pois as plaquetas não se aderem aos monômeros de FvW (5).

O processo de modificação é iniciado no retículo endoplasmático, onde ocorre a formação de pré-dímeros de FvW, que estão ligados entre si por pontes dissulfeto na porção carboxiterminal. No complexo de Golgi, outra ponte dissulfeto é acoplada, formando os dímeros. O grande multímeros de $\mathrm{FvW}$ (gmFvW) são sintetizados quando os dímeros formados se unem, possibilitando assim, o exercício de sua função no processo de coagulação (6-9).

Em 2001, Zheng et al. (5), descreveram a protease responsável pela clivagem destes multímeros e sua deficiência, um componente importante proveniente da família das metaloproteases, a ADAMTS13, cuja função é se ligar na porção carboxiterminal do gmFvW o clivando em unidades monoméricas, sendo a forma que não gera aderência de plaquetas devido ao ligante GPIb desse fator para a plaqueta não estar exposto nesse formato (4-9).

A glicoproteína Ib (CD 42) é uma glicoproteína com alta composição de leucina, presente na membrana plaquetária, grânulos alfa e denso plaquetários e no sistema de canalicular aberto (SNA), atuando como receptor para os gmFvW, além de receptor para a trombina. Após a ligação de ambos os componentes da castata de coagulação em seus respectivos receptores, ocorre a liberação dos grânulos plaquetários, contração plaquetária e maior potencial de agregação. O bom funcionamento da GPIb é fundamental para o controle do processo homeostático.

A ADAMTS13 tem como função principal manter a homeostase primária, sendo produzida principalmente no fígado. Com a clivagem oriunda desta protease, os gmFvW, que são seus multímeros, são reduzidos, contribuindo para que haja um equilíbrio entre diversos mecanismos, como a adesão plaquetária, seja em estímulo ou como barreira $(5,6)$.

Para ocorrer o processo de ativação, ela demanda de cátions bivalentes como $\mathrm{Zn}^{2+} \mathrm{e}$ $\mathrm{Ca}^{2+}$ para o funcionamento ideal enzimático. Ao realizar uma ligação proteolítica com o gmFvW gera uma redução de tamanho dos polímeros, mas não permanece ativa suficiente para que haja uma formação de uma trombose (4).

A deficiência da protease, perda da função, ou a produção de autoanticorpos anti-ADAMTS13 impede a clivagem dos gmFvW entre os aminoácidos tirosina e metionina, permitindo seu acúmulo nos vasos sanguíneos, dando início a sintomatologia correlata $(5,6)$.

A forma hereditária da doença possui caráter autossômico recessivo, ocorrendo uma mutação do gene da enzima ADAMTS13, localizado no cromossomo 9q34.2, levando a produção deficiente ou a ausência de ADAMTS13. É caracterizada por quadros 
recorrentes de trombocitopenia podendo estar acompanhado por isquemia de órgãos. Os pacientes estão sempre submetidos à recorrência, ocorrendo de forma irregular, na maioria dos casos (7).

A PTT adquirida é subdividida em imunomediada, quando ocorre a presença de autoanticorpos anti-ADAMTS13 e secundária, onde o excesso de estimulação das células endoteliais leva ao acúmulo de $\mathrm{gmFvW}$, dificultando a atividade da metaloprotease, que está em níveis reduzidos. Esse acúmulo ocorre devido a hiperplasia do endotélio, que ocasiona o crescimento excessivo do trombo (5-7). Neste caso, ocorre normalmente apenas um episódio agudo da doença (8).

Atualmente, há uma grande variedade de terapias que são utilizadas de forma adjacente à plasmaférese para garantir uma hemostasia adequada para estes pacientes. Dessa forma, este estudo busca abranger os principais recursos atuais utilizados juntamente a novas metodologias de tratamento que vem sendo abordadas.

\section{Material e métodos}

Foi realizada uma revisão da literatura no formato narrativo utilizando as bases de dados do PubMed, SciELO, Biblioteca Virtual em Saúde (BVS), Medline e o buscador Google Acadêmico nos idiomas português, espanhol e inglês.

Foram valorizadas as publicações que discorriam sobre terapias para o tratamento da PTT, bem como sua fisiopatologia em revisões, relatos de caso e artigos originais entre anos de 1982 a 2019, visando melhor fundamentação do tema. Usou-se as palavraschave plasmaférese, rituximabe, ADAMTS13 e caplacizumab, sendo descartados artigos que contemplassem outras púrpuras ou distúrbios microangiopáticos que não fossem a PTT, sendo incluídos 30 trabalhos ao final deste artigo.

\section{Achados clínicos e laboratoriais}

A pêntade descrita de sinais e sintomas é formada por febre, trombocitopenia, anemia hemolítica microangiopática, insuficiência renal e distúrbios neurológicos, porém, apenas em $40 \%$ dos casos é possível evidenciar a junção dos cinco elementos (10).

São relatadas ocorrências de epistaxe, gengivorragia, púrpuras e petéquias ao longo do corpo, dores difusas devido a isquemia causada pelo os microtrombos. trombose, icterícia, convulsões, fraqueza, arritmias, edemas e sintomas gastrointestinais (3).

O diagnóstico é feito com base na baixa contagem de plaquetas, a junção da pêntade de sintomas descrita no artigo, além de exames que excluam outras doenças hemostáticas que sejam ou não de caráter auto-imune. Os exames bioquímicos e o hemograma auxiliam a compreender a gravidade da hemólise e da vaso-oclusão ocasionada pela doença.

Estudos evidenciam que o endotélio dos vasos sanguíneos que irrigam os rins, cérebro, coração, pâncreas, baço e as glândulas suprarrenais são os mais afetados (11).

Observa-se no distendido sanguíneo a presença de esquizócitos oriundos da hemólise microangiopática, pontilhado basofílico, poiquilocitose e codócitos devido a diminuição da hemoglobina, estando em níveis inferiores a $10 \mathrm{~g} / \mathrm{dL}$. Ocorre reticulocitose, trombocitopenia com contagem inferior a $20.000 / \mathrm{mm}^{3}$, presença de eritroblastos e casualmente, megacariócitos (12). Analisando por microscopia óptica os microtrombos, nota-se uma aparência granular, sendo corados positivamente pelo Ácido Periódico de Shiff (PAS) (13-14).

Nos exames bioquímicos, nota-se o aumento da lactato desidrogenase (DHL) e da bilirrubina indireta. A diminuição da haptoglobina varia de acordo com a gravidade da 
hemólise. Em alguns casos, pode haver leucocitose com desvio à esquerda, porém, sem presença de hiato leucêmico ou outras anormalidades. O teste de Coombs é negativo e as provas da coagulação não apresentam alteração (13).

O teste de atividade da metaloprotease ADAMTS13 é realizado, tendo como resultado a ausência ou deficiência na função. Também pode ser realizado um ELISA para averiguar o nível de antígenos e anticorpos da ADAMTS13 nas amostras, além de poder medir sua atividade. Além destes testes de quantificação da enzima, o diagnóstico completo se dá pela avaliação do hemograma, principalmente para determinar a quantidade de plaquetas e se há presença de anemia hemolítica. Sempre deve ser realizado o diagnóstico diferencial de sepse, coagulação intravascular disseminada (CIVD), vasculite sistêmica e neoplasias disseminadas. A identificação da PTT de maneira rápida conduz um tratamento mais rápido e adequado, podendo utilizar a plasmaférese na fase inicial da doença, garantindo uma maior sobrevida do paciente $(12,13)$.

\section{Tratamentos}

A terapias para o PTT buscam ofertar ao paciente alternativas que melhorem seu quadro clínico, uma vez que não há cura para a doença. O uso de hemocomponentes vem sendo realizado como a administração de plasma com o intuito de corrigir a deficiência da ADAMTS13, possuindo elevada eficácia. O tratamento deve ser rapidamente inicializado visando um bom prognóstico da doença, impedindo que seja necessário intercorrências mais invasivas, como a esplenectomia.

Existem outros meios terápicos como a plasmaférese para retirada de gmFvW e autoanticorpos anti-ADAMTS13, pulsoterapia com corticoides visando a redução da produção de anticorpos antiplaquetários, utilização de anticorpos monoclonais quiméricos objetivando a diminuição da produção de autoanticorpos, e anticorpos específicos para FvW.

\section{Plasmaférese}

A aférese, de modo geral, consiste na retirada total do sangue, filtração, separação e devolução ao corpo de maneira automatizada. Neste processo, as células são infundidas junto ao líquido com características químicas similares ao plasma de volta ao organismo, enquanto ocorre a retirada do plasma. Esse processo é necessário para a correção de deficiência da ADAMTS13. O líquido utilizado na troca de plasma é preferencialmente o PFC, podendo ser utilizado o plasma isento de crioprecipitado (PIC), ou o plasma de 24h (P24h) (15).

Sua indicação é de primeira linha para PTT, sendo de caráter emergencial, devendo ser realizada de 24 a 36 horas após o diagnóstico clínico. O procedimento é realizado com coleta de amostra sanguínea para realização de exames e averiguação da condição do paciente pelo médico hemoterapeuta (16).

Inicialmente, o tratamento começa com uma única troca de volume plasmático $(40 \mathrm{~mL}$ $/ \mathrm{kg}$ de massa corporal) diariamente. A plasmaférese deve ser mantida até que os níveis de hemoglobina e de plaquetas estejam normalizados. É necessário avaliar a haptoglobina e DHL visando confirmar a estabilização da hemólise, sendo viável a transfusão de concentrado de hemácias (17).

Caso a frequência de uma plasmaférese ao dia não for suficiente, podem ser realizadas duas até a correção da sintomatologia. Porém, é necessário prosseguir com a terapia até cerca de uma a duas semanas após a normalização do quadro do paciente, A interrupção 
precoce ocasiona recorrência da doença em cerca de $40 \%$ a $50 \%$ dos pacientes, ocorrendo entre uma semana a um mês (18).

Para a terapêutica da PTT, a plasmaférese produz resultados satisfatórios, reduzindo a taxa de mortalidade de $90 \%$ para 10 a $30 \%$. Com o processo, há remoção dos autoanticorpos e citocinas responsáveis pelo dano no epitélio e uma melhora na deficiência da metaloprotease. Mas quando se trata de casos mais severos e com reincidência, o uso de outros tratamentos, como corticoides e imunoglobulinas, é altamente indicado (19).

\section{Líquidos repositores para a plasmaférese}

\section{Plasma Fresco Congelado (PFC)}

De acordo com o guia para uso de hemocomponentes do Ministério da Saúde (20), a infusão de PFC após a plasmaférese é recomendada como terapia inicial para o tratamento da PTT por ser fonte de ADAMTS13. As bolsas de PFC são reservadas exclusivamente para que atuem em casos de coagulopatias ativas. A plasmaférese terapêutica geralmente é realizada com reposição de outros líquidos, como albumina 3 a $5 \%$. Porém, não é aconselhável realizar a reposição apenas com albumina, podendo apresentar riscos de sangramento.

É constituído a partir da centrifugação do sangue total, sendo submetido ao congelamento até 8 horas após a coleta, pois alguns fatores de coagulação são lábeis. Fortemente indicado para o tratamento de coagulopatias. A dose terapêutica recomendada é de $10-15 \mathrm{~mL} / \mathrm{kg}(15)$.

De maneira geral, o plasma fresco congelado não é recomendado para deficiências hereditárias de fatores isolados. É utilizado apenas quando os produtos que possuem fatores de coagulação específicos não estão disponíveis. É indicado para correção de microangiopatias trombóticas, ou seja, a PTT e outras situações, como coagulação intravascular disseminada (CIVD), doenças hepáticas graves e deficiência de antitrombina (21).

Sua aplicação deve ser contínua, mesmo quando o paciente não está mais sobre a terapia da plasmaférese, atuando como líquido de suporte por gerir a metaloprotease necessária para a clivagem dos dímeros de FvW. Deve ser infundido em acesso venoso periférico, preferencialmente, durante 30 a 60 minutos (22).

\section{Plasma Isento de Crioprecipitado (PIC)}

O plasma isento de crioprecipitado (PIC), é resultado da retirada, em sistema fechado, do crioprecipitado, os separando em duas bolsas diferentes para o uso. Deve ser armazenado em $-18^{\circ} \mathrm{C}$. Não contém os fatores VIII, XIII, multímeros de von Willebrand e fibrinogênio, que estão disponíveis de maneira abundante nos crioprecipitados (17).

A única indicação para o uso do PIC é a PTT. Apesar de ser pobre para diversos fatores de coagulação, ainda apresenta valores altos da metaloprotease ADAMTS13, responsável pela metabolização do FvW (20). Seu uso clínico é em conjunto à plasmaférese, como líquido de reposição. Apesar de ser bem específica, não há estudos que comprovem que sua eficácia é maior que a do PFC (17).

Plasma de $24 h(P 24 h)$

É um hemocomponente derivado da mesma origem do PFC. É congelado entre 8 e 24 
horas após a coleta, sendo totalmente refrigerado. Apresenta redução de alguns fatores de coagulação, principalmente o V e VIII (20). O seu uso para tratamento de PTT aborda as mesmas questões do PFC e do PIC, apesar de não ser o concentrado utilizado em primeira instância (17).

Quadro 1 - Síntese dos pós e contras do uso da plasmaférese e líquidos repositores como terapia.

\begin{tabular}{|c|c|c|}
\hline Tipo de terapia & Pós & Contras \\
\hline Plasmaférese & $\begin{array}{c}\text { Seu uso reduz a taxa de } \\
\text { mortalidade de } 90 \% \text { para } 10 \text { a } \\
30 \% ; \\
\text { Corrige a deficiência de } \\
\text { ADAMST13; } \\
\text { Remove auto-anticorpos } \\
\text { patogênicos; } \\
\text { Em casos severos, pode ser } \\
\text { realizada uma intensificação } \\
\text { dos procedimentos de } \\
\text { plasmaférese; }\end{array}$ & $\begin{array}{c}\text { Não possui muita eficiência em } \\
\text { casos agudos e refratários, } \\
\text { demanda terapia adicional; }\end{array}$ \\
\hline Plasma Fresco Congelado & \begin{tabular}{|c|} 
Possui indicação para \\
tratamento de microangiopatias \\
trombóticas em um geral; \\
Tratamento de primeira linha \\
para PTT;
\end{tabular} & \begin{tabular}{|c|} 
Não é indicado para casos \\
hereditários; \\
Não deve ser transfundido em \\
pacientes em terapia intensiva; \\
Possui riscos de desenvolver \\
efeitos adversos;
\end{tabular} \\
\hline $\begin{array}{l}\text { Plasma Isento de } \\
\text { Crioprecipitado }\end{array}$ & $\begin{array}{l}\text { Por não conter os FVW, é } \\
\text { utilizado em casos de PTT } \\
\text { refratária; } \\
\text { Contribui para remissão } \\
\text { completa; }\end{array}$ & $\begin{array}{c}\text { Seu uso é apenas em conjunto } \\
\text { com a plasmaférese, não possui } \\
\text { utilidade clínica para PTT } \\
\text { individualmente; } \\
\text { Não possui evidência de ter } \\
\text { melhor ação do que o PFC; }\end{array}$ \\
\hline Plasma de 24 horas & $\begin{array}{c}\text { Utilizado para as mesmas } \\
\text { indicações clínicas que o PFC; } \\
\text { Fácil obtenção; }\end{array}$ & $\begin{array}{c}\text { Redução dos fatores V e VIII } \\
\text { da coagulação; } \\
\text { Não possui evidência de ter } \\
\text { melhor ação do que o PFC; }\end{array}$ \\
\hline
\end{tabular}

Fonte: Sadler (3); Brasil (20).

\section{Anticorpos}

\section{Rituximabe}

O rituximabe, de acordo com a ANVISA (23), é um anticorpo monoclonal quimérico (camundongo/humano) que se liga de forma específica ao antígeno CD20 que se expressa desde os linfócitos pré-B até os linfócitos B maduros. Ao se ligar, inicia-se o processo de lise celular, impedindo a formação de anticorpos, consequentemente, bloqueando a produção de autoanticorpos anti-ADAMTS13. Um fato a se considerar é a alta especificidade do rituximabe, não atingindo as células progenitoras, pró-B, plasmócitos, e células de outros tecidos.

Foi evidenciado em artigos e relatos de caso que o uso do medicamento teve bom prognóstico em 93\% dos casos (24). Outros estudos, como de Fakhouri (25) demonstrou 
grande eficácia quando usado em tratamento da PTT refratária a plasmaférese. Foi analisado seis pacientes que receberam quatro doses semanais de rituximabe. Dois deles estavam no primeiro episódio da doença, em plasmaférese por um mês e, havia sido administrado o antimitótico vincristina. A total melhora do quadro clínico ocorreu entre 5 e 14 dias após a quarta dose de rituximabe.

O estudo de George (24) contribuiu para elucidar a melhora clínica dos pacientes que sofrem de PTT por produção de autoanticorpos. Nele é demonstrado que esses pacientes podem ter uma mortalidade mais alta mesmo com o uso de glicocorticoides, e que o uso do rituximabe como tratamento coadjuvante confere benefícios ao paciente.

\section{Caplacizumab}

É um anticorpo bivalente de domínio único desenvolvido especificamente para o tratamento da PTT, inibindo a interação entre o FvW e o receptor de glicoproteína IbIX-V presente nas plaquetas, evitando a adesão plaquetária mediada pelo os gmFvW. O campo de ação também diminui a produção do fator VIII e os níveis do FvW durante o tratamento. $\mathrm{O}$ medicamento diminui a agregação plaquetária induzida pela ristocetina (RIPA) para $10 \%$ e o cofator de ristocetina (RICO) para $20 \%$.

Um estudo realizado por Scully (26) analisou 145 pacientes, onde alguns receberam o medicamento durante a troca plasmática e outros receberam placebo durante um período de 30 dias. O tempo para a normalização das plaquetas foi mais rápido com o uso do Caplacizumab do que com o placebo, com intervalo de 2,96 dias, sendo que os pacientes que receberam o Caplacizumab possuíam $1,55 \mathrm{x}$ mais chances de normalizar a contagem de plaquetas.

A porcentagem de pacientes que apresentaram recorrência de PTT foi $67 \%$ menor com o medicamento do que com placebo, além de permanecerem menos tempo hospitalizados e precisarem de menos trocas plasmáticas.

\section{Corticoides}

O tratamento com corticoides tem o intuito de reduzir a produção de anticorpos antiplaquetários. A pulsoterapia com metilprednisolona é realizada em quadros exacerbados da doença, visando a diminuição da inflamação e estabilização da crise. A imunossupressão auxilia nos casos de PTT adquirida auto-imune (25).

Apesar de a plasmaférese juntamente ao uso de corticoides ser um tratamento fortemente indicado, não apresenta bom prognóstico em pacientes refratários. Porém, existem estudos que demonstram que doses diárias de prednisona podem ter respostas favoráveis, especialmente em pacientes com sintomatologia leve e sem queixas neurológicas (18). 
Quadro 2 - Síntese dos pós e contras do uso de fármacos como terapia adicional a plasmaférese.

\begin{tabular}{|c|c|c|}
\hline Tipos de terapia & Pós & Contras \\
\hline Rituximabe & $\begin{array}{c}\text { Impede a formação de auto- } \\
\text { anticorpos anti-ADAMTS13; } \\
\text { Oferece alta especificidade, } \\
\text { diminuindo os efeitos adversos; } \\
\text { Demonstrou bom prognóstico em } \\
\text { 93\% dos casos; } \\
\text { Apresenta bons resultados em } \\
\text { pacientes refratários; }\end{array}$ & $\begin{array}{l}\text { Poucos estudos abordando o uso } \\
\text { direcionado ao tratamento da PTT; }\end{array}$ \\
\hline Caplacizumab & $\begin{array}{c}\text { Inibe a interação entre o FvW e o } \\
\text { receptor de glicoproteína Ib-IX-V } \\
\text { presente nas plaquetas, evitando a } \\
\text { adesão plaquetária mediada pelo } \\
\text { os gmFvW; } \\
\text { Terapia desenvolvida } \\
\text { especificamente para a PTT; }\end{array}$ & $\begin{array}{l}\text { Pouco conhecimento acerca dos } \\
\text { efeitos adversos por estar há pouco } \\
\text { tempo em comercialização, sendo } \\
\text { aprovado em } 2018 \text { na União } \\
\text { Européia e em } 2019 \text { nos EUA; }\end{array}$ \\
\hline Corticóides & $\begin{array}{l}\text { Apresenta boa resposta quando } \\
\text { associado à plasmaférese, sendo } \\
\text { considerado tratamento de } \\
\text { primeira linha; }\end{array}$ & $\begin{array}{c}\text { Grupos de pacientes refratários } \\
\text { não apresentam resposta ao } \\
\text { tratamento. }\end{array}$ \\
\hline
\end{tabular}

Fonte: Sadler (3), BRASIL (20), Georde et al. (24), Fakhouri et al. (25) e Scully et al. (26).

\section{Esplenectomia}

Foi relatado que a esplenectomia leva à remissão em pacientes com PTT que não possuíram melhora do quadro com a plasmaférese ou àqueles que tiveram recidiva (27), porém, é desaconselhável em gestantes. Estudos mostram que ocorre uma melhora de $50 \%$ ou mais nos casos recidivados (28). De forma geral, teoricamente, o uso da esplenectomia possui benefícios devido ao baço ser um órgão vinculado a hematopoese e ao sequestro de plaquetas, mas há pouca evidência sobre sua eficácia clínica e segurança.

\section{Tratamentos contra-indicados}

Não é recomendada a transfusão de concentrado de plaquetas e administração de medicamentos que estimulem a produção de trombopoietina, visto que agravam os quadros trombóticos, salvo em casos de sangramento acentuado, onde a vida do paciente esteja em risco. Foi relatado que infusão de plaquetas está vinculada a alterações no sistema nervoso central pela alta taxa de agregação plaquetária (29).

Antiplaquetários, como a ticlopidina e clopidogrel, apesar de interessantes para o tratamento dos microtrombos de agregados plaquetários, não são amplamente aceitos na literatura, uma vez que podem aumentar o risco de sangramento ou até mesmo, possibilitar um episódio de PTT adquirida imunomediada (30).

\section{Conclusão}

Analisando os estudos mencionados no artigo em questão, é possível concluir que atualmente, as terapias possuem significado clínico de grande importância. Diversos tratamentos vêm sendo utilizados, aumentando o espectro de ação e melhora do quadro do paciente. É importante observar que os dados atuais sobre o uso de anticorpos monoclonais quiméricos e bivalente de domínio único apresentam resultados benéficos quando comparados apenas ao uso da plasmaférese concomitante aos corticoides, tendo 
uma boa perspectiva de eficácia de tratamento.

Deve ser ressaltado que a qualidade da plasmaférese está vinculada ao tipo de líquido repositor utilizado, sendo o PFC o mais recomendado. É importante evidenciar que mesmo após a resolução do episódio de crise da PTT, é necessário prosseguir com os exames laboratoriais de rotina para acompanhamento, visto que em alguns casos, os episódios podem se tornar recorrentes.

Ainda assim, nenhum tratamento ainda é completamente eficaz. O paciente portador da PTT deve estar sempre submetido a sessões de plasmaférese com reposição líquida até atingir um quadro de remissão estável, de pelo menos três dias consecutivos, onde deve estar normalizado os níveis de hemoglobina, haptoglobina, DHL e contagem de plaquetas. Também, quando necessário, deve utilizar os anticorpos e outros fármacos para alcançar um fim de episódios agudos da doença.

\section{Referências}

1. Retornaz F, Durand JM, Poullin P, Lefèvre P, Soubeyrand J. Le purpura thrombotique thrombocytopénique idiopathique ou syndrome de Moschowitz: actualités physiopathologiques et perspectives thérapeutiques. La Revue de medicine interne 2000; 21(9):777-784.

2. Pessegueiro P, Pires C. Síndrome hemolítico urémico/púrpura trombocitopénica trombótica. Medicina Interna 2005; 12(2): 102-116.

3. Sadler JE. Thrombotic thrombocytopenic purpura: a moving target. Hematology Am Soc Hematol Educ Program 2006: 415-20.

4. Moake JL, Rudy CK, Troll JH, Weinstein MJ, Colannino NM, Azocar J, et al. Unusually large plasma factor VIII:von Willebrand factor multimers in chronic relapsing thrombotic thrombocytopenic purpura. N Eng J Med 1982; 307 (23): 1432-5.

5. Zheng X, Chung D, Takayama TK, Majerus EM, Sadler JE, Fujikawa K. Structure of von Willebrand factor-cleaving protease (ADAMTS13), a metalloprotease involved in thrombotic thrombocytopenic purpura. J Biol Chem 2001; 276(44): 41059-63.

6. Furlan M, Robles R, Galbusera M, Remuzzi G, Kyrle PA, Brenner B, et al. Von Willebrand factorcleaving protease in thrombotic thrombocytopenic purpura and the hemolytic-uremic syndrome. $\mathrm{N}$ Engl J Med 1998; 339(22): 1578-84.

7. Tsai HM. Molecular mechanisms in thrombotic thrombocytopenic purpura. Semin Thromb Hemost 2004; 30(5): 549-57.

8. Klaus C, Plaimauer B, Studt JD, Dorner F, Lämmle B, Mannucci PM, et al. Epitope mapping of ADAMTS13 autoantibodies in acquired thrombotic thrombocytopenic purpura. Blood 2004; 103(12): 4514-9.

9. Anstadt MP, Carwile JM, Guill CK, Conklin LD, Soltero ER, Lucci A, Kroll MH. Relapse of thrombotic thrombocytopenic purpura associated with decreased VWF cleaving activity. Am J Med Sci 2002; 323(5): 281-4.

10. Bandarenko N, Brecher ME. United States Thrombotic Thrombocytopenic Púrpura Apheresis Group (US TTP ASG): multicenter survey and retrospective analysis of current efficacy of therapeutic plasma exchange. J Clin Apheresis 1998; 13(3): 133-41.

11. De la Hunt MN, Morris KP, Coulthard MG, Rangecroft L. Oesophageal and severe gut involvement in the haemolytic uraemic syndrome. Br J Surg 1991; 78(12): 1469-72.

12. Mann KG. Biochemistry and pathology of blood coagulation. Thromb Haemost. 1999; 82: 165-74.

13. Sadler JE, Moake JL, Miyata T, George JN. Recent advances in thrombotic thrombocytopenic purpura. Hematology. American Society Hematol Educ Program, 2004: 307-23.

14. Kwaan HC. Clinicopathologic features of thrombotic thrombocytopenic purpura. Semin Hematol 1987; 24(2): 71-81

15. Brasil, Ministério da Saúde. Portaria n 158, de 4 de Fevereiro de 2016. Brasília, 2016. Acessível em: <http://bvsms.saude.gov.br/bvs/saudelegis/gm/2016/prt0158_04_02_2016.html>.

16. Szczepiorkowski ZM, Winters JL, Bandarenko N et al. Guidelines on the use of therapeutic apheresis 
in clinical practice evidence-based approach from the apheresis applications committee of the American Society for Apheresis. Journal of Clinical Apheresis 2010; 25(3): 83-177.

17. Nabhan C, Kwaan HC. Current concepts in the diagnosis and management of thrombotic thrombocytopenic purpura. Hematol Oncol Clin North Am 2003; 17: 177-99.

18. Bell WR, Braine HG, Ness PM et al. Improved survival in thrombotic thrombocytopenic purpurahemolytic uremic syndrome. Clinical experience in 108 patients. N Engl J Med 1991; 325(6): 398403.

19. Brasil, Ministério da Saúde. Brasília, 2005. Manual de tratamento das coagulopatias hereditárias. Acessível

em: http://www.saude.pr.gov.br/arquivos/File/MANUAISHEMEPAR/ManualdetratamentodasCOAGUL OPATIASHEREDITaRIAS.pdf.

20. Brasil, Ministério da Saúde, Departamento de Atenção Especializada, guia para o uso de Hemocomponentes. Brasília, 2015. Acessível em: http://bvsms.saude.gov.br/bvs/publicacoes/guia_uso_hemocomponentes_2ed.pdf

21. Miller R, Eriksson LI, Flesher LA, Wiener-Kronish JP. Miller's Anesthesia. 7th ed. Philadelphia: Elsevier; 2009. p. 1739-66

22. Hemocentro Campinas, Unicamp, Universidade Estadual de Campinas. Campinas, 2018. Acessível em: https://www.hemocentro.unicamp.br/arquivos/2018/09/Manual-deOrienta\%C3\%A7\%C3\%B5es-em-Hemoterapia-2018.pdf.

23. Anvisa, Agência Nacional de Vigilância Sanitária. Rituximabe. Acessível em: http://portal.anvisa.gov.br/informacoes-

tecnicas 13?p_p_id=101_INSTANCE_WvKKx2fhdjM2\&p_p_col_id=column-

2\&p_p_col_pos=1\&p_p_col_count=2\&_101_INSTANCE_WvKKx2fhdjM2_groupId=219201\&_10

1_INSTANCE_WvKKx2fhdjM2_urlTitle=mabthera-rituximabe-nova-

indicacao\&_101_INSTANCE_WvKKx2fhdjM2_struts_action=\%2Fasset_publisher\%2Fview_conten t\&_101_INSTANCE_WvKKx2fhdjM2_assetEntryId=5670217\&_101_INSTANCE_WvKKx2fhdjM 2_type $=$ content

24. George JN, Woodson RD, Kiss JE, Kojouri K, Vesely SK. Rituximab therapy for thrombotic thrombocytopenic purpura: a proposed study of the Transfusion Medicine/Hemostasis Clinical Trials Network with a systematic review of rituximab therapy for immunemediated disorders. J Clin Apher 2006; 21(1):49-56.

25. Fakhouri F, Vernant JP, Veyradier A, Wolf M, Kaplanski G, Binaut R et al. Efficiency of curative and prophylactic treatment with rituximab in ADAMTS13-deficient thrombotic thrombocytopenic purpura: a study of 11 cases. Blood 2005;106(6): 1932-7.

26. Scully M, Cataland SR, Peyvandi F, Coppo P, Knöbl P, Kremer Hovinga JA, Metjian A, de la Rubia J, Pavenski K, Callewaert F, Biswas D, De Winter H, Zeldin RK; HERCULES Investigators. Caplacizumab treatment for acquired thrombotic thrombocytopenic purpura. N Engl J Med 2019; 380(4): 335-346.

27. Hoffkes HG, Weber F, Uppenkamp M, et al. Recovery by splenectomy in patients with relapsed C. Nabhan, H.C. Kwaan / Hematol Oncol Clin N Am 17 (2003) 177-199 197 thrombotic thrombocytopenic purpura and treatment failure to plasma exchange. Semin Thromb Hemost 1995; 21(2): 161-5.

28. Winslow GA, Nelson EW. Thrombotic thrombocytopenic purpura: indications for and results of splenectomy. Am J Surg 1995; 170(6): 558-63.

29. Gordon LI, Kwaan HC, Rossi EC. Deleterious effects of platelet transfusions and recovery thrombocytosis in patients with thrombotic microangiopathy. Semin Hematol 1987; 24(3): 194-201.

30. Rosove MH, Ho WG, Goldfinger D. Ineffectiveness of aspirin and dipyridamole in the treatment of thrombotic thrombocytopenic purpura. Ann Intern Med 1982; 96(1): 27-33. 